\title{
On the Propagation Properties of Surface Waves
}

\author{
V. Jakšić ${ }^{1}$, S. Molchanov ${ }^{2}$, L. Pastur ${ }^{3}$ \\ ${ }^{1}$ Institute for Mathematics and its Applications \\ University of Minnesota, 514 Vincent Hall \\ 55455-0436 Minneapolis \\ Minnesota, U.S.A. \\ ${ }^{2}$ Mathematics Department, University of North Carolina \\ Charlotte, NC, U.S.A. \\ ${ }^{3}$ Institute of Low Temperature Physics \\ Academy of Sciences of Ukraine \\ Kharkov, Ukraine
}

\section{Introduction}

Surface waves were discovered by Rayleigh at the end of the last century [1]. He considered a homogeneous and isotropic elastic halfspace $R_{+}^{3}=$ $\left\{(x, \xi), x \geq 0, \xi \in R^{2}\right\}$, whose boundary surface $x=0$ is free of traction. He discovered that there are two types of solutions of the respective boundary value problem:

(i) Solutions which are oscillating and nondecaying in all variables. They are called the volume (bulk) waves. 
(ii) Solutions which are the plane waves in the longitudinal variables $\xi$ and which are exponentially decaying in the transverse variable $x$. This solutions are called the surface (grazing) waves. They propagate only in the longitudinal directions, with the velocity slightly smaller than the velocity of the volume waves.

The similar solutions exist if the plane $x=0$ is the interface between the two halfspaces with different elastic constants (see $[1,2]$ for references and discussion). This should be compared to the case when a homogeneous elastic body occupies the whole $R^{3}$ and when the solutions are the plane waves in all variables.

The Rayleigh result is perhaps the first demonstration of a rather general property of solutions of differential and finite-difference equations which can be summarized as: If the coefficients of the equations are strongly inhomogeneous (spatially dependent), then the equations may have solutions which are localized near the inhomogeneities.

Returning to the surface waves, we remark that after the Rayleigh discovery the similar solutions for the Maxwell equations were found, at the turn of the century, by the Sommerfeld school in the study of the propagation properties of the radio waves around the earth surface. These are the electromagnetic waves that propagate along the surface of a dielectric subspace or the interface between the two halfspaces with different dielectric constants and decay exponentially with the increase of the distance from surface (intersurface). These solutions are now known as the surface polaritons or the surface plasmons (the latter correspond to the limiting case $c=\infty$, where $c$ is the velocity of light) [3].

The natural analogue of the Rayleigh problem, in the case of inhomogeneous (and in particular, randomly inhomogeneous) media, is a model of an inhomogeneous elastic halfspace. The common wisdom of the localization theory suggests that in the case of a randomly inhomogeneous elastic medium occupying the whole space $R^{d}, d \geq 3$, delocalized (extended) states 
exist for low and high frequencies, and localized states exist for an interval ("window") of intermediate frequencies (if the disorder is large enough). If so, then it is natural to expect that in the case of half-infinite random inhomogeneous media the above picture should be complemented by the surface solutions which are delocalized (propagating) with respect to the transverse coordinates, if the inhomogeneity is weak enough (or if their frequency is small enough).

The above picture assumes the positive solution of a hard problem, the proof of the existence of delocalized states in a randomly inhomogeneous media. We will consider in this note a class of simpler problems where similar phenomena is expected to emerge. A typical example is the boundary value problem for the Laplace equation:

$$
-\Delta_{X} u=E u, \quad X=(x, \xi) \in R_{+}^{d}=\left\{x \geq 0, \xi \in R^{d-1}\right\}
$$

with the boundary condition

$$
\left.\frac{\partial u}{\partial x}\right|_{x=0}=V(\xi) u(0, \xi), \xi \in R^{d-1} .
$$

If $V(\xi)$ is a constant, $V(\xi) \equiv a$, then the eigenvalue problem can be solved by separation of the variables. Its solutions can be explicitly identified as follows.

(i) If $a>0$ then the solutions are

$$
u(X, K)=\left(2^{d-2} \pi^{d}\left(k^{2}+a^{2}\right)\right)^{-1 / 2} e^{i \varphi \xi}(k \cos k X+a \sin k X)
$$

where $K=(k, \varphi) \in R^{d}, E=k^{2}+\varphi^{2} \geq 0$.

(ii) If $a<0$ then we have two classes of solutions:

$$
u_{1}(X, K)=\left(2^{d-2} \pi^{d}\left(k^{2}+a^{2}\right)\right)^{-1 / 2} e^{i \varphi \xi}(k \cos k X+a \sin k X)
$$

where $K=(k, \varphi) \in R^{d}, E=k^{2}+\varphi^{2}>0$, and

$$
u_{2}(X, \varphi)=\left(2^{d} \pi^{d-1}|a|\right)^{-1 / 2} e^{i \varphi \xi-|a| x}
$$


where $\varphi \in R^{d-1}, E=\varphi^{2}-a^{2} \geq-a^{2}$

Thus for $a<0$ we have analogues of the volume and the surface waves; the only difference between (1.1)-(1.2) and the Rayleigh problem is that the spectral parameter can be negative.

This note is an introduction to the program whose goal is to understand the structure of the solutions of the eigenvalue problem (1.1)-(1.2) in which $V$ is a quasi-periodic or random function. In the next section we give a more precise description of the program; the explicitly solvable model $V(\xi) \equiv$ const will serve us as a quide. In the Section 3 we present some rigorous results concerning the discrete analog of the model (1.1)-(1.2).

\section{Generalities}

We begin by reformulating the boundary value problem (1.1)-(1.2) in terms of spectral theory.

Let us recall that the spectrum of an abstract selfadjoint operator $H$ consists of the absolutely continuous, singular continuous and pure point components: $\sigma(H)=\sigma_{a c}(H) \bigcup \sigma_{s c}(H) \bigcup \sigma_{p p}(H)$. Typically, the given operator $H$ is a differential or finite difference operator and it is generally accepted that generalized eigenfunctions corresponding to $\sigma_{a c}(H)$ describe the propagating waves and particles. A typical example is the Schrödinger operator in $L^{2}\left(R^{d}\right)$, whose potential decays at infinity. If this decay is fast enough, then the absolutely continuous spectrum of this operator is $R_{+}$; the respective eigenfunctions are superpositions of the incident plane waves and scattered spherical waves (the Sommerfeld solutions).

The boundary value problem (1.1)-(1.2) defines the selfadjoint operator $H_{V}$ acting in the space $L^{2}\left(R_{+}^{d}\right)$. Thus, we can reformulate the results (1.3)$(1.5)((V(\xi) \equiv a)$ as follows:

(i) $a>0$. The spectrum of $H_{V}$ is $R_{+}$and is purely absolutely continuous. The respective eigenfunctions are given by (1.3); they are the plane 
waves with respect to the longitudinal coordinates $\xi \in R^{d-1}$ and the standing waves with respect to the transverse coordinate $x \geq 0$. This system of eigenfunctions is orthonormal and complete, i.e.

$$
\begin{aligned}
\int_{R_{+}^{d}} u\left(X, K_{1}\right) u\left(X, K_{2}\right) d X & =\delta\left(K_{1}-K_{2}\right) \\
\int_{R^{d}} u\left(X_{1}, K\right) u\left(X_{2}, K\right) d K & =\delta\left(X_{1}-X_{2}\right)
\end{aligned}
$$

(ii) $a<0$. The spectrum of $H_{V}$ is the interval $\left[-a^{2}, \infty\right) \supset R_{+}$, and is again purely absolutely continuous. The generalized eigenfunctions are given by (1.4) and (1.5); we call them respectively the volume (bulk) and the surface (grazing) solutions (waves). These eigenfunctions satisfy the relations:

$$
\begin{aligned}
& \int_{R_{+}^{d}} u_{1}\left(X, K_{1}\right) u_{1}\left(X, K_{2}\right) d X=\delta\left(K_{1}-K_{2}\right), \\
& \int_{R_{+}^{d}} u_{2}\left(X, \varphi_{1}\right) u_{2}\left(X, \varphi_{2}\right) d X=\delta\left(\varphi_{1}-\varphi_{2}\right), \\
& \int_{R_{+}^{d}} u_{1}(X, K) u_{2}(X, \varphi) d X=0 \\
& \int_{R^{d}} u_{1}\left(X_{1}, K\right) u_{1}\left(X_{2}, K\right) d K+\int_{R^{d-1}} u_{2}\left(X_{1}, \varphi\right) u_{2}\left(X_{2}, \varphi\right) d \varphi=\delta\left(X_{1}-X_{2}\right) .
\end{aligned}
$$

Thus, the volume waves $\left\{u_{1}(X, K)\right\}_{K \in R^{d}}$ and the surface waves $\left\{u_{2}(X, \varphi)\right\}_{\varphi \in R^{d-1}}$ generate two orthogonal subspaces. In other words, the spectrum of $H_{V}$, for $V(\xi) \equiv a<0$, consists of two "layers" (channels) $[0, \infty)$ and $\left[-a^{2}, \infty\right)$ corresponding to volume waves (1.4) and surface waves (1.5). There is no scattering between the volume channel and the surface channel.

We now mention a few other problems that have similar structure of the spectrum.

The Schrödinger operator in $R^{d}$ with a surface potential. 
We consider the Schrödinger equation

$$
H u=-\Delta_{X} u+2 \delta(x) V(\xi) u=E u
$$

on $R^{d}=\left\{X=(x, \xi) \mid x \in R, \xi \in R^{d-1}\right\}$, where $\delta(x)$ is the Dirac $\delta$-function. For simplicity, we assume that the surface potential $V(\xi)$ is bounded. Let

$$
G_{z}(X \mid Y)=(H-z)^{-1}(X \mid Y), \quad g_{z}(X-Y)=(-\Delta-z)^{-1}(X \mid Y),
$$

for the complex spectral parameter $z, \operatorname{Im} z \neq 0$. The Green formula yields

$$
G_{z}(X \mid Y)=g_{z}(X-Y)-2 \int_{R^{2}} g_{z}(x, \zeta-\xi) V(\zeta) G_{z}(0, \xi \mid Y) ;
$$

setting $x=0$ in this formula we obtain an integral equation for $G_{z}(0, \zeta \mid Y)$ :

$$
G_{z}(0, \zeta \mid Y)=g_{z}(y, \xi-\eta)-2 \int_{R^{2}} g_{z}(0, \xi-\zeta) V(\zeta) G_{z}(0, \zeta \mid Y),
$$

On the other hand, the Green formula for the Green function of the problem (1.1)-(1.2), and the Green function

$$
g_{z}^{(N)}(X \mid Y)=g_{z}(x-y, \xi-\eta)+g_{z}(x+y, \xi-\eta)
$$

of the Neumann problem (corresponding $V=0$ in (1.2)) yield the same integral equation since

$$
g_{z}^{(N)}(0, \xi \mid 0, \eta)=2 g_{z}(0, \xi-\eta)
$$

Thus the spectral problems (1.1)-(1.2) and (2.6) are equivalent.

Discrete boundary value problem. On the half-space

$$
Z_{+}^{d}=\left\{X=(x, \xi), x \in[0, \infty), \xi \in Z^{d-1}\right\}
$$

we consider the spectral problem

$$
u(x-1, \xi)+u(x+1, \xi)+\left(\Delta_{d-1} u\right)(x, \xi)=E u, x \geq 0
$$




$$
u(-1, \xi)=V(\xi) u(0, \xi)
$$

There

$$
\left(\Delta_{d-1} u\right)(\xi)=\sum_{\eta \in Z^{d-1},|\xi-\eta|=1} u(\eta)
$$

is the discrete Laplacian in $Z^{d-1}$. This boundary value problem is the natural discrete analogue of (1.1)-(1.2).

Discrete Schrödinger operator with a surface potential. The respective operator is defined by the finite-difference equation in

$$
\begin{gathered}
Z^{d}=\left\{(x, \xi), x \in Z, \xi \in Z^{d-1}\right\} ; \\
-\Delta_{d} u+2 \delta(x) V(\xi) u=E u
\end{gathered}
$$

where $\delta(x)$ is the Kronecker $\delta$-symbol.

Discrete Schrödinger operator with the "subspace" potential. We decompose $Z^{d}$ as

$$
Z^{d}=Z^{d_{1}} \times Z^{d_{2}}=\left\{X=(x, \xi), x \in Z^{d_{1}}, \xi \in Z^{d_{2}}\right\}
$$

and consider the finite-difference equation

$$
-\Delta_{d} u+\delta(x) V(\xi) u=E u
$$

where

$$
\delta(x)=\prod_{j=1}^{d_{1}} \delta\left(x_{j}\right)
$$

The potential is now concentrated on the subspace $Z^{d_{2}}$. If $d=3, d_{1}=1$, $d_{2}=2$ this model can be regarded as a model of the thin film; for $d=3$, $d_{1}=2, d_{2}=1$ it can be regarded as a model of the line inhomogeneity. We call the latter case the polymer problem. The case $d=3, d_{1}=1, d_{2}=2$ and $V$ depending only on $\xi_{1}$ reduces to $d=2, d_{1}=d_{2}=1$ and can be regarded as a model of the grating (the linear interferometer). 
It is easy to show that all these problems with $V=$ const have the surface (subspace) solutions which decay exponentially as $|x| \rightarrow \infty$ and which are the plane waves in $\xi$-variable, i.e. propagate along the subspace $Z^{d_{2}}$.

Similar results are also known for the case when $V(\xi)$ is periodic (see $[5-7])$.

The goal of our program is to analyze the spectral and scattering properties of the above models, in the cases when the function $V(\xi)$ is quasi-periodic and random; to analyze the structure of the generalized eigenfunctions of the models and to study their propagation properties; to understand to what extent the simple division of spectra into the "volume" channel and "surface" channel persists in the models after replacing the constant boundary condition with a function of the above type.

For the rest of this note we will discuss only the discrete boundary value problem. We present certain rigorous result on the model in the next section. To give the reader the taste of the results we are aiming to, we finish this section with the following simple result. Recalling the property of polynomial boundedness of generalized eigenfunctions of finite-difference operators we define the set $S$ of the surface (subspace) solutions for the model (2.10) by

$$
S=\left\{u_{E}(x, \xi), \sup _{\xi \in Z^{d_{2}}}\left(1+|\xi|^{a}\right)^{-1} \sum_{x \in Z^{d_{1}}}\left|u_{E}(x, \xi)\right|^{2}<\infty\right\}
$$

where $a>d$ is fixed. We also introduce

$$
\sigma_{S}=\overline{\left\{E: u_{E} \in S\right\}}
$$

Proposition 2.1 Let $H_{0}=\Delta_{d}$ and let $H_{V}$ be the selfadjoint operator defined by (2.10). Let $\sigma\left(H_{V}\right)$ and $\sigma\left(H_{0}\right)=\sigma_{a c}\left(H_{0}\right)=[-2 d, 2 d]$ be their spectra. Then $\sigma\left(H_{V}\right) \backslash \sigma\left(H_{0}\right) \subset \sigma_{S}$ and the corresponding generalized eigenfunctions decay exponentially as $|x| \rightarrow \infty$.

The proof of this proposition follows from: 
(i) the Green formula

$$
u_{E}(x, \xi)=\sum_{\eta \in Z^{d_{2}}} g_{E}(x, \xi-\eta) V(\eta) u_{E}(0, \eta)
$$

(ii) the polynomial bound

$$
\left|u_{E}(X)\right| \leq C_{\varepsilon}\left(1+|X|^{d / 2+\varepsilon}\right), \quad \varepsilon>0,
$$

which is valid for almost all $E$ with respect to the spectral measure of $H_{V}$;

(iii) the exponential decay of the "free" Green function $g_{E}(X), E \notin$ $\sigma\left(H_{0}\right)$.

\section{Discrete boundary value problem}

In this section we present some rigorous results concerning the discrete boundary value problem (2.7)-(2.9) introduced in Section 2. We would like to emphasize in advance that our understanding of the problem is limited; we have only a few results to announce here. We plan to present a more complete analysis of the problem in [4].

Set $V(\xi)=g v(\xi)$ where $v(\xi), \xi \in Z^{d-1}$, are independent, identically distributed random variables with continuous density $p(v)$. The parameter $g$ measures the strength of the coupling between waves (quantum particles) and the random corrugated surface of the medium. The boundary value problem (2.7)-(2.9) can be alternatively defined as the spectral problem for the operator

$$
\begin{aligned}
& H_{V} u(x, \xi)=u(x+1, \xi)+u(x-1, \xi)+\sum_{\left|\xi-\xi^{\prime}\right|=1} u\left(x, \xi^{\prime}\right), \quad \text { if } x \geq 1, \\
& H_{V} u(0, \xi)=u(1, \xi)+\sum_{\left|\xi-\xi^{\prime}\right|=1} u\left(0, \xi^{\prime}\right)+g v(\xi) u(0, \xi) .
\end{aligned}
$$

Here the subscript $V$ stands for the usual random parameter (random realization of the potential), see e.g. [10]. The case in which the random 
potential $v(\xi)$ is nonzero only along certain parts of the boundary $\partial Z_{+}^{d}$ is of the independent interest; for the sake of brevity we will not discuss such models here.

In the case when the random variables $v(\xi)$ are degenerate, $v(\xi) \equiv a$, the spectrum of the operator $H_{V}$ is absolutely continuous and fills the union of two intervals

$$
\sigma\left(H_{V}\right)=[-2 d, 2 d] \cup\left[-2(d-1)+E_{0}(g a), 2(d-1)+E_{0}(g a)\right]
$$

there $E_{0}(g a)=g a+[g a]^{-1},\left|E_{0}(g a)\right| \geq 2$, is the only eigenvalue of the one dimensional boundary value problem $u(x-1)+u(x+1)=E u(x)$, $u(-1)=\operatorname{gau}(0)$ (this eigenvalue exists iff $|g a|>1$ ). As in the continuous case, these intervals correspond to two "channels", volume waves and surface waves. If the surface channel exists $(|g a|>1)$, then it has a "tail" lying outside of $\sigma\left(H_{0}\right)$. The generalized eigenfunction associated to the "volume channel" do not decay in any direction; the ones associated to the the "surface channel" are exponentially decaying in the $x$-variable.

Our goal is to understand how is the structure of spectrum affected after the replacement of the constant potential along the boundary with the random one.

The standard ergodicity argument (see e.g. [9], or [10]), yields that there are closed sets $\Sigma_{a c}, \Sigma_{p p}, \Sigma_{a c} \subset R$ so that for a.e. $V, \sigma_{a c}\left(H_{V}\right)=\Sigma_{a c}, \sigma_{s c}\left(H_{V}\right)=$ $\Sigma_{s c}, \sigma_{p p}\left(H_{V}\right)=\Sigma_{p p}$. In particular, for a.e. $V$

$$
\sigma\left(H_{V}\right)=\Sigma_{a c} \cup \Sigma_{s c} \cup \Sigma_{p p} \equiv \Sigma
$$

In fact, it is not too difficult to explicitly identify the set $\Sigma$. If $\mathcal{V}$ is the support of density function $p(v)$, namely the closure of the set $\{v: p(v) \neq 0\}$, then

$$
\Sigma=[-2 d, 2 d] \cup\left\{[-2(d-1), 2(d-1)]+E_{0}(g \mathcal{V})\right\}
$$

where $X+Y=\{x+y, x \in X, y \in Y\}$. 
It is a characteristic feature of the model that the operator $H_{V}$ always has lots of absolutely continuous spectra, due to the free propagation along the $x$-axis.

Theorem 3.1 If $\int_{R}|v| p(v) d v<\infty$ then $\Sigma_{a c} \supset[-2 d, 2 d]$.

The basic idea of the proof is to show that there is a dense set of vectors $\mathcal{D} \subset l^{2}\left(Z_{+}^{d}\right)$ so that the limit

$$
s-\lim _{t \rightarrow \infty} \exp \left(i t H_{V}\right) \exp \left(-i t H_{0}\right) u, \quad u \in \mathcal{D},
$$

exists, see e.g. [16]. By the Cook criterion, it suffices to show that

$$
\int_{1}^{\infty}\left\|\left(H_{V}-H_{0}\right) \exp \left(-i t H_{0}\right) u\right\| d t<\infty
$$

for each $u \in \mathcal{D}$. Clearly, (3.12) will follow if

$$
\sum_{\xi \in Z^{d-1}}|v(\xi)|\left|\left(\delta_{n}, \exp \left(-i t H_{0}\right) u\right)\right|<\infty,
$$

for almost all $V$ and all $u \in \mathcal{D}$. One establishes (3.13) by passing first to the Fourier variables in the $\xi$-variables (see the discussion below), and then using integration by parts, judicious choice of the set $\mathcal{D}$, and Borel-Cantelli lemma. The details will be presented in [4].

Further discussion of the spectral properties of $H_{V}$ is based on the Fourier transformed form of $H_{V}$. The operator $H_{V}$ is unitarily equivalent to operator $\hat{H}_{V}$ which acts on $l^{2}\left(Z^{+}\right) \otimes L^{2}\left(T^{d-1}\right)$. We denote the variables on that space by $(x, \phi)=\left(x, \phi_{1}, \phi_{2}, \ldots \phi_{d-1}\right)$, and its elements by $\hat{u}(x, \phi)$. Let

$$
\Phi(\phi)=2 \sum_{i=1}^{d-1} \cos \left(\phi_{i}\right) .
$$

The operator $\hat{H}_{V}$ is given by

$$
\begin{aligned}
\hat{H}_{V} \hat{u}(x, \phi) & =\hat{u}(x+1, \phi)+\hat{u}(x-1, \phi)+\Phi(\phi) \hat{u}(x, \phi), \quad x \geq 1, \\
\hat{H}_{V} \hat{u}(0, \phi) & =\hat{u}(1, \phi)+g\left[\widehat{v_{V} u}\right](0, \phi) .
\end{aligned}
$$


It is not too difficult to show that integrable solutions of the equation

$$
\hat{H}_{V} \hat{u}(x, \phi)=E \hat{u}(x, \phi)
$$

have the form

$$
\hat{u}(x, \phi)=\hat{u}(0, \phi) \Lambda_{\phi, E}^{x} ;
$$

the function $\Lambda_{\phi, E}$ is determined by the relations

$$
\Lambda_{\phi, E}+\frac{1}{\Lambda_{\phi, E}}+\Phi(\phi)=E \quad \text { and } \quad 0<\Lambda_{\phi, E}<1 .
$$

In particular, if $E \in[-2 d, 2 d]$, we may assume that the function $\hat{u}(0, \phi)$ is supported on the set

$$
\left\{\phi \in T^{d-1}:|\Phi(\phi)-E|>2\right\} .
$$

It follows from (3.14) that function $\hat{u}(0, \phi)$ satisfies the equation

$$
\hat{u}(0, \phi) \Lambda_{\phi, E}^{-1}=-g\left[\widehat{v_{V} u}\right](\phi) .
$$

This equation will play the central role in the subsequent discussion. We recall that the set $\mathcal{V}$ is support of the density function $p(v)$. Our first application of (3.15) is:

Proposition 3.2 a) Suppose that $\mathcal{V} \subset[-a, a]$ for some $a>0$. If $|g|<1 / a$ then $H_{V}$ has no eigenvalues in $[-2 d, 2 d]$.

b) Suppose that $\mathcal{V} \subset[a, b]$ for some constants $0<a<b$. If $g>2(2 d-1) / a$ then $H_{V}$ has no eigenvalues on $[-2 d, 2 d]$.

Remark. The above results are in fact deterministic.

Remark. Suppose that $\mathcal{V}=[-a, a]$. Then the part a) yields that there are no eigenvalues on $[-2 d, 2 d]$ as long as there is no spectrum outside the spectrum of Laplacian. The part b) yields that as long as the spectrum outside $[-2 d, 2 d]$ is separated from the one in $[-2 d, 2 d]$ by a sufficiently large 
gap, then are no embedded eigenvalues in $[-2 d, 2 d]$.

Proof: The equation (3.14) yields that

$$
\left.\int_{T^{d-1}}|\hat{u}(0, \phi)|^{2}\left|\Lambda_{\phi, E}^{-1}\right|^{2} d \phi=|g| \int_{T^{d-1}} \mid \widehat{v_{V} u}\right]\left.(0, \phi)\right|^{2} d \phi .
$$

Assume that $u$ is normalized as

$$
1=\int_{T^{d-1}}|\hat{u}(0, \phi)|^{2} d \phi=\sum_{\xi \in Z^{d-1}}|u(0, \xi)|^{2} .
$$

Since

$$
1 \leq \Lambda_{\phi, E}^{-1} \leq 2(2 d-1)
$$

we get from (3.14) that

$$
1 \leq g^{2} \sum_{n \in Z^{d-1}}|\xi(x, \omega)|^{2}|u(0, n)|^{2} \leq[2(2 d-1)]^{2} .
$$

The result is immediate.

We now turn to the analysis of the structure of the "tail" part of the spectrum of $H_{V}$, given by $\Sigma \backslash[-2 d, 2 d]$. Let $R_{V}\left(X, X^{\prime} ; E+i \epsilon\right)$ be the matrix elements of the resolvent $\left(H_{V}-z\right)^{-1}$ for $z=E+i \epsilon$. Let $X_{0} \equiv\left(0, \xi_{0}\right)$ be fixed point on the boundary $\partial Z_{+}^{d}$ and let $|E|>2 d$. Then

$$
\hat{R}_{V}\left(X_{0},(x, \phi) ; E+i \epsilon\right)=\hat{R}_{V}\left(X_{0},(0, \phi) ; E+i \epsilon\right)\left[\Lambda_{\phi, E+i \epsilon}\right]^{x}
$$

There $\Lambda_{\phi, E+i \epsilon}$ is the analytic continuation of the function $\Lambda_{\phi, E}$ from the part of the real axis $|E|>2 d$ to the upper half-plane $\{w \mid \operatorname{Im}(w)>0\}$. For $\xi \in Z^{d-1}$ let $D(\xi, E+i \epsilon)$ be the inverse Fourier transform of $\Lambda_{\phi, E+i \epsilon}$. Obviously, for each $|E|>2 d$ there is $C_{E}>0$ and $\gamma(E)>0$ so that

$$
\sup _{\epsilon>0}\left|D_{E}(\xi, E+i \epsilon)\right|<C_{E} \exp (-\gamma(E)|\xi|) .
$$

The resolvent equation restricted to the boundary $\partial Z_{+}^{d}$ becomes

$$
\left[D\left(\xi-\xi_{0}, E+i \epsilon\right)+\Delta_{d-1}+g v(\xi)\right] R_{V}\left(\left(0, \xi_{0}\right),(0, \xi) ; E+i \epsilon\right)=
$$




$$
=\left(\delta\left(\xi-\xi_{0}\right)+E+i \epsilon\right) R_{V}\left(\left(0, \xi_{0}\right),(0, \xi) ; E+i \epsilon\right) .
$$

On this way we have obtained a $(d-1)$-dimensional eigenvalue problem which, however, depends non-linearly on the spectral parameter $E$. Nevertheless, the techniques developed in the spectral theory of random operators can be properly adapted to handle the problem in the strong localization regime and we have (see [11],[12])

Theorem 3.3 Suppose that density $p(v)$ satisfies $\sup _{v \in R}|p(v)|<\infty$. Then for $\forall \delta>0$ there is $g(\delta)>0$ so that the estimate

$$
\sup _{\epsilon>0}\left|R_{V}\left(\left(0, \xi_{0}\right),(0, \xi) ; E+i \epsilon\right)\right|<C_{E, \xi_{0}, V} \exp \left(-\gamma(E)\left|\xi-\xi_{0}\right|\right),
$$

holds for $|g|>g(\delta)$, each fixed $|E|>2 d+\delta$ and for a.e. $V$. The same estimate holds for each fixed $g$ if $|E|$ is taken large enough, $|E|>E_{0}(g)$.

Remark. The result holds under more general condition on density $p(v)$. Remark. Naturally, the constant $\gamma(E)$ in (3.17) may differ from the one in Theorem 3.3. For notational simplicity, we will always use the letter $\gamma(E)$ for the $E$-dependent constant figuring in the exponential decay of the quantity in question.

From the estimate (3.18) and relation (3.16) it follows that under the conditions of the Theorem 3.3, the resolvent kernel of $H_{V}$ satisfies

$$
\sup _{\epsilon>0}\left|R_{V}\left(X_{0}, X ; E+i \epsilon\right)\right| \leq C_{V, X_{0}, E} \exp \left(-\gamma(E)\left|X_{0}-X\right|_{+}\right),
$$

in the large coupling/high energy regime described in the Theorem 3.3. The Simon-Wolff's theorem [9], [10]) yields the following result:

Theorem 3.4 Under the conditions of Theorem 3.3 we have:

a) For each $\delta>0$ there is $g(\delta)>0$ so that for $|g|>g(\delta)$,

$$
\Sigma \cap\{E|| E \mid \geq 2 d+\delta\} \subset \Sigma_{p p}
$$


The corresponding eigenfunctions decay exponentially.

b) For each $g \neq 0$ there is $E(g)$ so that

$$
\Sigma \cap\{E|| E \mid \geq E(g)\} \subset \Sigma_{p p}
$$

The corresponding eigenfunctions decay exponentially.

Consider now the quasiperiodic potential

$$
V(\xi)=g \tan \pi[(\alpha, \xi)+\omega]
$$

where $\alpha=\left(\alpha_{1}, . . \alpha_{d-1}\right)$ is a Diophantine vector, i.e.

$$
|(\alpha, \xi)+\omega| \geq C|\xi|^{\beta}
$$

for all $\xi \in Z^{d-1} \backslash\{0\}, m \in Z$ and some positive $C$ and $\beta ; \omega \in[0,1]$ is the "randomness" parameter.

The potential (3.19) can model a quasiperiodically (strongly) corrugated surface. The Schrödinger operator with a similar potential is analyzed in [10]. This operator has pure point spectrum coinciding with $R$ for all $g \neq 0$ and almost all $\omega \in[0,1]$ with respect to the Lebesgue measure.

Similarly, for the surface potential (3.19) we have [13]:

Theorem 3.5 Let $H_{V}$ be the operator defined by (2.7)-(2.9) and (3.19). Then the spectrum of $H_{V}$ is $R$ and its part lying outside $\sigma\left(H_{0}\right)=[-2 d, 2 d]$ is pure point for almost all $\omega \in[0,1]$ with respect to the Lebesgue measure. The eigenvalues are simple and dense on $R \backslash[-2 d, 2 d]$ and the corresponding eigenfunctions decay exponentially in $\xi$.

Combining Proposition 2.1 (which naturally also holds for the model (2.7)(2.9)) and Theorems 3.4 and 3.5 we conclude that in the strong localization regime the eigenfunctions are the surface solutions of respective equation and that they decay exponentially not only in the transverse coordinates $x$ bur 
also in the longitudinal coordinates $\xi$. In other words, in the cases treated in Theorems 3.4 and 3.5 the surface waves are localized by strong fluctuations of the random potential.

On the other hand, according to Theorem 3.1, the absolutely continuous spectrum fills the interval $[-2 d, 2 d]$ for all strengths of the coupling. Thus we are naturally lead to the following questions:

(I) Is the spectrum of $H_{V}$ purely absolutely continuous for $g$ small?

(II) What is the nature of the spectrum on the interval $[-2 d, 2 d]$ for the intermidate and large values of $g$ ? Do we have embedded eigenvalues in a.c. spectrum?

(III) Do surface solutions exist on the interval $[2 d, 2 d]$ ? Are they propagating? What are the respective conditions?

Concerning (I), if $d=3$ and the random potential is placed only along a line (the polymer case), then the conjecture can be established using a version of Kato's smooth perturbation theory [4]. Concerning (III), the answer is affirmative if $d=3$ and i.i.d. random variables $v\left(\xi_{1}, \xi_{2}\right)$ do not depend on $\xi_{2}$. Indeed, in this case the dependence of solutions on $\xi_{2}$ is harmonic and the corresponding energies belong to the absolutely continuous spectrum of $H_{V}$. On the other hand, since the analogue of the respective non-linear spectral problem (3.15) is one-dimensional, one might hope to obtain some informations modifying the existing techniques of 1-d random Schrödinger operator theory. In [4] we modify and extend the technique developed in [14], [15], to prove (for the model (2.7)-(2.9)) that if $d=2$ then under general conditions and for any $g$ we have $\Sigma \cap\{E:|E| \geq 4\} \subset \Sigma_{p p}$. This allows us to prove that if $v\left(\xi_{1}, \xi_{2}\right)$ does not depend on $\xi_{2}$ then $\sigma_{S} \cap[-6,6] \neq \emptyset$. The corresponding solutions propagate along $\xi_{2}$-axis and exponentially decay in $\xi_{1}$ and $x$. 


\section{References}

[1] J.W.S. Rayleigh. The theory of sound. Dover, N.Y. 1945

[2] A.E.Love. A Treatise on the Mathematical Theory of Elasticity. Dover, N.Y. 1944

[3] V.Agranovich and D.Mills (eds) Surface Polaritons. North Holland, 1982.

[4] V. Jakšić, S. Molchanov, L. Pastur (in preparation)

[5] E.Davies, B.Simon. Commun.Math.Phys. $\underline{\underline{63}}$ (1978) 277-301

[6] A.Grossman, R.Hoegh-Krohn, M.Mebkkout. Commun.Math.Phys. $\underline{\underline{77}}$ (1980) $87-110$

[7] Yu. Karpeshina. Theor. Math. Fyz. $\underline{\underline{57}}$ (1983) 304-313

[8] E.Englisch, M. Schroder, P.Seba. Ann.Inst. H. Poincare. $\underline{\underline{46}}$ (1987) 373382

[9] R.Carmona, J. Lacroix. Spectral Theory of Random Schrodinger Operators. Birkhauser, Boston, 1992

[10] L.Pastur, A.Figotin. Spectra of Random and Almost Periodic Operators. Springer Verlag, Heidelberg 1992

[11] V.Grinshpun. Dopovidi Akademii Nauk Ukrainy (Proc. Ac. Sci. of Ukraine). $\underline{\underline{8}}$ (1992) 18-21; $\underline{\underline{9}}$ (1993) 26-29

[12] M.Aizerman, S.Molchanov. Commun.Math.Phys. 157 (1993) 245-279

[13] B.Khoruzhenko, L.Pastur (unpublished) (1993) 
[14] W.Kirsch, S.Molchanov, L.Pastur. Funct. Analysis and its appl. $\underline{\underline{24}}$ (1990) $14-25$

[15] S.Molchanov. Lecture Notes in Math. 1581 (1994), Springer Verlag, Heidelberg 1994.

[16] T. Kato. Perturbation Theory for Linear Operators. Springer Verlag, Heidelberg, 1966. 